\title{
Perfil das mulheres submetidas à inserção do dispositivo intrauterino de cobre na Atenção Primária à Saúde de municípios da Paraíba
}

Profile of women submitted to insertion of the intrauterine copper device in the Primary Health Care of municipalities of Paraíba Perfil de las mujeres sometidas a la inserción del dispositivo intrauterino de cobre en la Atención Primaria a la Salud de municipios de Paraíba

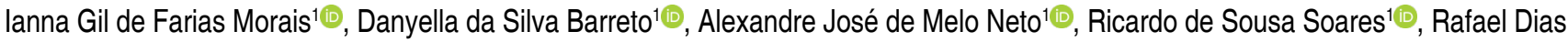
Gonçalves $^{1}{ }^{\circledR}$, Maria Eduarda de Melo Pereira do Rêgo ${ }^{1}{ }^{\oplus}$, Priscylla Silveira Ramos Costa ${ }^{10}$

${ }^{1}$ Universidade Federal da Paraíba (UFPB), João Pessoa, PB, Brasil.

\section{Resumo}

Introdução: Dentre os contraceptivos reversíveis de longa ação disponíveis no mercado, o dispositivo intrauterino (DIU) de cobre é o método oferecido pelo Ministério da Saúde para ser disponibilizado na rede de serviços do Sistema Único de Saúde (SUS). Apesar de ser uma forma de contracepção com alta eficácia e segurança, inclusive em adolescentes e nulíparas, o DIU ainda é subutilizado no Brasil. Objetivo: Traçar o perfil das mulheres submetidas à inserção de DIU de cobre na Atenção Primária à Saúde (APS) de municípios da Paraíba. Métodos: Estudo transversal, descritivo, com amostra de 246 mulheres submetidas à inserção de DIU de cobre em Unidades Básicas de Saúde (UBS) de municípios da Paraíba entre 2016 e 2019. Informações sobre idade, ocupação, escolaridade, estado civil e paridade dessas mulheres foram coletadas de prontuários médicos e registros do procedimento. As variáveis nominais foram apresentadas por meio de suas frequências absolutas e relativas e as variáveis contínuas por meio de suas médias, medianas e modas. Resultados: A idade das mulheres variou de 15 a 50 anos, sendo a idade média de 27,4 anos, 158 (64,2\%) usuárias eram casadas ou estavam em união consensual e 150 (61\%) mulheres apresentavam pelo menos o ensino médio completo. Na amostra, $110(44,7 \%)$ mulheres exerciam alguma atividade remunerada e $97(39,4 \%)$ declararam-se do lar. Foram observadas 27 (11\%) nulíparas e $215(87,4 \%)$ mulheres com pelo menos um filho, sendo uma média de 1,6 filhos por mulher. Dentre as adolescentes do estudo, $15(75 \%)$ tinham pelo menos um filho. Conclusão: A maioria das mulheres que inseriram DIU na APS de municípios da Paraíba apresentava entre 20 e 29 anos, possuía ensino médio completo, exercia atividade remunerada e continha de 1 a 2 filhos. As baixas porcentagens de mulheres com baixa escolaridade, adolescentes e nulíparas encontradas na amostra apontam para a necessidade de traçar estratégias específicas de incentivo ao dispositivo intrauterino na Atenção Primária à Saúde.

Palavras-chave: Perfil de Saúde; Dispositivos Intrauterinos; Atenção Primária à Saúde.

Como citar: Morais IGF, Barreto DS, Melo Neto AJ, Soares RS, Gonçalves RD, Rêgo MEMP, Costa PSR. Perfil das mulheres submetidas à inserção do dispositivo intrauterino de cobre na Atenção Primária à Saúde de municípios da Paraíba. Rev Bras Med Fam Comunidade. 2021;16(43):2649. https://doi.org/10.5712/rbmfc16(43)2649

\author{
Autor correspondente: \\ lanna Gil de Farias Morais. \\ E-mail: ianna_gil@ hotmail.com \\ Fonte de financiamento: \\ próprio. \\ Parecer CEP: \\ CAAE: 09941619.2.0000.8069 \\ Procedência: \\ não encomendado. \\ Avaliação por pares: \\ externa. \\ Recebido em: 25/07/2020. \\ Aprovado em: 02/04/2021.
}




\begin{abstract}
Introduction: Among the long-acting reversible contraceptives available on the market, the copper intrauterine device (IUD) is the method offered by the Brazilian government to be made available through the Unified Health System (SUS). Despite being a form of contraception with high efficacy and safety, including in adolescents and nulliparous, the IUD is still underutilized in Brazil. Objectives: To trace the profile of women submitted to the insertion of copper IUDs in Primary Health Care of municipalities in the state of Paraiba. Methods: This was a descriptive cross-sectional study with a sample of 246 women submitted to copper IUD insertion in the Primary Health Care of municipalities of Paraíba between 2016 and 2019. Information on age, occupation, education, marital status, and parity of these women were collected from medical records and records of the procedure. The nominal variables were presented by means of their absolute and relative frequencies and the continuous variables by means of their means, medians, and mode. Results: The age of women ranged from 15 to 50 years, with a mean age of 27.4 years, $158(64.2 \%)$ were married or in consensual union and $150(61 \%)$ women had at least complete. In the sample, $110(44.7 \%)$ women were engaged in some paid activity and $97(39.4 \%)$ declared housewife. There were $27(11 \%)$ nulliparous and $215(87.4 \%)$ women with at least one child, with an average of 1.6 children per woman. Among the adolescents in the study, 15 (75\%) had at least one child. Conclusion: Most of the women who inserted IUDs in the Primary Health Care of Paraiba were between 20 and 29 years of age, had completed high school, had paid work, and had 1 to 2 children. The low percentages of women with low levels of education, adolescents and nulliparas found in the sample point to the need to outline specific strategies to encourage intrauterine devices in Primary Health Care.
\end{abstract}

Keywords: Health Profile; Intrauterine Devices; Primary Health Care.

\title{
Resumen
}

Introducción: Entre los anticonceptivos reversibles de larga acción disponibles en el mercado, el dispositivo intrauterino (DIU) de cobre es el método ofrecido por el gobierno brasileño para estar disponible en la red de servicios del Sistema Único de Salud (SUS). A pesar de ser una forma de contracepción con alta eficacia y seguridad, incluso en adolescentes y nulíparas, el DIU aún es infrautilizado en Brasil. Objetivo: Trazar el perfil de las mujeres sometidas a la inserción de DIU de cobre en la Atención Primaria a la Salud de municipios del estado de Paraíba. Métodos: Estudio transversal, descriptivo, con muestra de 246 mujeres sometidas a la inserción de DIU de cobre en la Atención Primaria a la Salud de municipios de Paraíba entre 2016 y 2019. Información sobre edad, ocupación, educación, estado civil y paridad de esas mujeres fueron recolectadas de prontuarios médicos y registros del procedimiento. Las variables nominales fueron presentadas por medio de sus frecuencias absolutas y relativas y las variables continuas por medio de sus medias, medianas y modas. La edad de las mujeres varía de 15 a 50 años, la edad promedio siendo de 27,4 años, $158(64,2 \%)$ usuarias estaban casadas o estaban en unión consensual y $150(61 \%)$ mujeres terminaron por al menos sus estudios de preparatoria. En la encuesta, 110 (44,7\%) mujeres ejercían alguna actividad remunerada y $97(39,4 \%)$ eran amas de casa. Se observaron $27(11 \%)$ nulíparas y $215(87,4 \%)$ mujeres con al menos un hijo, la media siendo de 1,6 hijos por mujer. Entre las adolescentes del estudio, 15 (75\%) tenían al menos un hijo. Conclusión: La mayoría de las mujeres que ingresaron DIU en la Atención Primaria a la Salud en el estado Paraíba eran de las edades entre 20 y 29 años, poseía enseñanza media completa, ejercía actividad remunerada y tenían entre de 1 a 2 hijos. Los bajos porcentajes de mujeres con baja educación, adolescentes y nulíparas encontradas en la encuesta indican la necesidad de trazar estrategias específicas de incentivo al dispositivo intrauterino en la Atención Primaria a la Salud.

Palabras clave: Perfil de Salud; Dispositivos Intrauterinos; Atención Primaria de Salud.

\section{Introdução}

O direito reprodutivo representa um conceito recentemente desenvolvido, reconhecido em leis nacionais e conferências internacionais. Consiste no direito das pessoas optarem por terem ou não filhos, com autonomia para escolherem quantos e quando tê-los, livre de discriminação, imposição e violência, tendo seu exercício assegurado por políticas públicas. ${ }^{1}$ Segundo Piovesan², refere-se ao direito de autodeterminação, privacidade, intimidade, liberdade e autonomia individual, com dimensão típica dos direitos civis.

Apesar dos avanços constitucionais referentes ao planejamento reprodutivo, a porcentagem de gestações não intencionais aumentou em todas as sub-regiões da América Latina, indo de 59\% nos anos de 1990 a 1994 para 69\% de 2010 a 2014. ${ }^{3}$ Dados da última Pesquisa Nacional de Demografia e Saúde (PNDS), realizada em 2006, revelam que 54\% das mulheres haviam planejado a gravidez e $18 \%$ das gestações eram indesejadas. ${ }^{4}$ Entre 2011 e 2012, a prevalência de gravidez não planejada chegava a 55,4\% no Brasil. ${ }^{5}$

Adifusão dos métodos contraceptivos apresenta impacto positivo na saúde da mulher ${ }^{6}$ e os contraceptivos reversíveis de longa ação, conhecidos como LARC (Long Acting Reversible Contraceptive) representam uma importante estratégia custo-efetiva na prevenção de gestações indesejadas pois são métodos com alta eficácia, aproximadamente 20 vezes mais eficazes do que pílulas, adesivos ou anéis ${ }^{7-10}$. Entre os LARCS, o DIU de cobre é o método ofertado pelo Sistema Único de Saúde (SUS) que age na redução da motilidade e viabilidade espermática ${ }^{11,12}$. 
O DIU apresenta taxas de falha no primeiro ano de $0,6 \%$ a $0,8 \%{ }^{13}$ e apresenta uma retomada da fertilidade imediata após a retirada em média de $84,75 \%{ }^{14}$. O método é ofertado seguramente a maioria das mulheres incluindo adolescentes e nulíparas ${ }^{15-19}$, podem ser utilizados no pós parto e pós aborto ${ }^{20-21}$, possui uma taxa de continuação de $85 \%$ em um ano com efeitos colaterais já conhecidos ${ }^{22-24}$. Mesmo com todas as vantagens já conhecidas, o DIU é subutilizado no Brasil com taxa de $1,9 \%{ }^{25}$ e diversas barreiras organizacionais foram identificadas para sua ampliação ${ }^{26}$

Estudo realizado em três capitais brasileiras mostrou o perfil das mulheres que tinham interesse em colocar o DIU mas ainda não inseriram. As mulheres que tinham entre 18 e 24 anos, com 12 anos ou mais de escolaridade, não unidas, com plano de saúde, com filhos e com maior nível de conhecimento sobre o DIU foram as que mais expressaram o interesse em usá-lo ${ }^{27}$.

Diante disso, faz-se nacessária a caracterização das mulheres que inseriram o DIU APS pois, essa informação pode auxiliar na construção de estratégias de incentivo ao DIU em grupos com elegibilidade, porém ainda com baixa adesão a esse dispositivo na APS. Nesse contexto, o objetivo desse estudo é traçar o perfil das mulheres submetidas à inserção do DIU de cobre em 13 UBS distribuídas entre quatro municípios da Paraíba.

\section{Métodos}

Trata-se de um estudo transversal, descritivo, realizado em treze UBS distribuída em quatro municípios diferentes da Paraíba (Conde, Sapé, Caaporã e João Pessoa), cenários da atuação de projetos de extensão da UFPB, que promovem ações educativas e práticas de treinamento para inserção do DIU na APS que foi idealizado para superar umas das principais barreiras encontradas na literatura 27, que é a falta de conhecimento dos profissionais quanto a técnica de inserção.

O projeto de extensão realiza uma visita à UBS que deseja receber o treinamento para apresentar os critérios de elegibilidade e as etapas do treinamento. Cabe ao projeto levar a equipe formada por médicos de família e comunidade responsável pelo treinamento e cabe às UBS organizar toda a infraestrutura e seleção das mulheres com realização prévia de anamnese e exame ginecológico. Após o treinamento, as equipes seguem inserindo na rotina da UBS e registrando os dados em um caderno com dados semelhantes em todas as UBS pois o objetivo é gerar autonomia no processo. Com o projeto de extensão, UBS treinadas passaram a ter essa oferta em sua carta de serviços.

Incluiu-se no estudo todo o universo de mulheres que, sob livre demanda e obedecendo aos critérios de elegibilidade propostos pela Organização Mundial de Saúde (OMS), inseriram o DIU nessas UBS entre junho de 2016 e maio de 2019.

Os dados referentes ao perfil das mulheres submetidas à inserção do DIU (idade, ocupação, escolaridade, estado civil e paridade) foram coletados a partir de prontuários médicos e, principalmente, de cadernos de registro do procedimento em questão, instrumentos deixados nas UBS após as ações extensionistas de treinamento profissional, a fim de terem dados sociodemográficos das mulheres e dados do procedimento preenchidos pelo médico na consulta de inserção do DIU.

Os dados coletados foram digitados no software Excel para construção de um banco de dados estruturado. Os dados anonimizados estarão disponíveis mediante solicitação à autora correspondente e apresentação de projeto de pesquisa com aprovação por comitê de ética. Para a análise dos dados, tal banco foi exportado para o software SPSS (Statistical Package of Social Science), recodificando-se algumas variáveis iniciais em variáveis 
dicotômicas e numéricas para melhor utilizá-las na análise, tomando por base dados já presentes na literatura. Na análise descritiva, as variáveis nominais foram apresentadas por meio de suas frequências absolutas e relativas e as variáveis contínuas por meio de suas médias, medianas e modas.

O projeto de pesquisa recebeu aprovação do Comitê de Ética em Pesquisa com Seres Humanos (CEP/CCM/ UFPB) em março de 2019, conforme parecer $n^{\circ} 3.239 .798$, sendo considerados os aspectos éticos da pesquisa envolvendo seres humanos, preconizados pela Resolução 466/12 do Conselho Nacional de Saúde (CNS). A população não participou do planejameto ou condução da pesquisa. O trabalho não obteve financiamento externo.

\section{Resultados}

Foram analisadas características referentes ao perfil sociodemográfico e à paridade de 246 mulheres submetidas à inserção do DIU de cobre, demonstradas na Tabela 1.

Tabela 1 . Perfil das mulheres submetidas à inserção de DIU de cobre

\begin{tabular}{|c|c|c|}
\hline Variável & $\mathbf{N}$ & $(\%)$ \\
\hline \multicolumn{3}{|l|}{ Idade (anos) } \\
\hline $15 \mid-20$ & 20 & 8 \\
\hline $201-25$ & 75 & 30 \\
\hline $25 \mid-30$ & 71 & 29 \\
\hline $30 \mid-35$ & 39 & 16 \\
\hline $35 \mid-40$ & 27 & 11 \\
\hline $40 \mid-45$ & 8 & 3 \\
\hline $45 \mid-50$ & 4 & 2 \\
\hline$\geq 50$ & 1 & 0,4 \\
\hline Não informada & 1 & 0,4 \\
\hline Idade Média (anos): 27,4 & Mediana: 26 & Moda: 25 \\
\hline \multicolumn{3}{|l|}{ Estado civil } \\
\hline Casada & 104 & 42 \\
\hline Solteira & 61 & 25 \\
\hline União consensual & 54 & 22 \\
\hline Divorciada ou separada & 4 & 2 \\
\hline Não informado & 23 & 9 \\
\hline \multicolumn{3}{|l|}{ Escolaridade } \\
\hline Fundamental incompleto & 22 & 9 \\
\hline Fundamental completo & 18 & 7 \\
\hline Médio incompleto & 27 & 11 \\
\hline Médio completo & 103 & 42 \\
\hline Superior incompleto & 19 & 8 \\
\hline Superior completo & 28 & 11 \\
\hline Não informado & 29 & 12 \\
\hline \multicolumn{3}{|l|}{ Ocupação } \\
\hline Dolar & 97 & 39 \\
\hline Outra ocupação & 135 & 55 \\
\hline Desempregada & 3 & 1 \\
\hline Não informada & 11 & 5 \\
\hline \multicolumn{3}{|l|}{ Paridade } \\
\hline 0 & 27 & 11 \\
\hline 1 & 98 & 40 \\
\hline 2 & 80 & 32 \\
\hline$\geq 3$ & 37 & 15 \\
\hline Não informada & 4 & 2 \\
\hline Paridade Média (anos): $\quad 1,562$ & Mediana: 1 & Moda: 1 \\
\hline Total & 246 & 100 \\
\hline
\end{tabular}


Aidade das mulheres variou de 15 a 50 anos, sendo a idade média de 27,4 anos e a moda encontrada de 25 anos. Na amostra em questão, apenas 20 (8\%) mulheres estavam na faixa etária de 15 a 19 anos, enquanto 146 (59\%) tinham 20 a 29 anos.

Quanto ao estado civil, $158(64 \%)$ usuárias eram casadas ou estavam em união consensual. Em relação ao nível educacional, 150 (61\%) mulheres apresentavam pelo menos o ensino médio completo e $29(11,8 \%)$ não apresentaram dados referentes à escolaridade registrados.

Além disso, $110(44,7 \%)$ mulheres exerciam alguma atividade remunerada, enquanto 97 (39,4\%) declararam-se do lar, 25 (10\%) estudantes e $3(1 \%)$ desempregadas. Dentre as ocupações remuneradas especificadas, a profissão de professora foi a que apresentou maior frequência na amostra, sendo referida por 16 mulheres $(6,5 \%)$.

Em relação aos antecedentes obstétricos, verificou-se uma média aproximada de 1,6 filhos por mulher, moda de 1,0 filho e faixa de paridade de 0 a 5 filhos, com $215(87,4 \%)$ mulheres com pelo menos um filho e 27 (11\%) nulíparas.

Diante da observância da expressiva porcentagem de mulheres que se declararam do lar e da pequena representatividade de adolescentes e nulíparas entre as mulheres que inseriram DIU de cobre na APS da Paraíba, optou-se pela análise das características específicas desses grupos.

As características das adolescentes (15 a 19 anos) que inseriram o DIU estão descritas na Tabela 2. Constatou-se que 9 (45\%) delas estavam casadas ou em união consensual, 9 (45\%) eram solteiras, 6 (30\%) tinham pelo menos ensino médio completo, 15 (75\%) delas tinham um ou mais filhos. A média encontrada de filhos no grupo das adolescentes foi de 1,0. Em relação à ocupação exercida, $8(40 \%)$ declararam-se do lar e 9 (45\%) estudantes e apenas 3 (15\%) referiram atividade remunerada.

Tabela 2 . Perfil das adolescentes submetidas à inserção de DIU de cobre

\begin{tabular}{lcc}
\hline Variável & N & (\%) \\
\hline Estado civil & 9 & 45 \\
$\quad$ Solteira & 3 & 15 \\
Casada & 6 & 30 \\
União consensual & 1 & 5 \\
Divorciada ou separada & 1 & 5 \\
$\quad$ Não informado & & \\
Escolaridade & 3 & 15 \\
$\quad$ Fundamental incompleto & 4 & 20 \\
Fundamental completo & 6 & 30 \\
Médio incompleto & 6 & 30 \\
Médio completo & 0 & 0 \\
Superior incompleto & 0 & 0 \\
Superior completo & 1 & 5 \\
Não informado & & \\
Paridade & 5 & 25 \\
$\quad 0$ & 11 & 55 \\
1 & 3 & 15 \\
2 & 1 & 5 \\
$\geq 3$ & Mediana: 1,0 & Moda:1,0 \\
Paridade Média (anos): 1,0 & 20 & 100 \\
Total & & \\
\hline
\end{tabular}


Ao analisar o perfil das nulíparas submetidas à inserção do DIU (Tabela 3), verificou-se uma idade média de 24 anos e que 18 (66\%) delas declararam-se solteiras. Além disso, foi observado que 21 (78\%) apresentavam pelo menos ensino médio completo. Quanto à ocupação exercida, 12 (44\%) eram estudantes, 7 (26\%) apresentavam profissão especializada e apenas 4 (14\%) declararam-se do lar.

Tabela 3 . Perfil das nulíparas submetidas à inserção de DIU de cobre

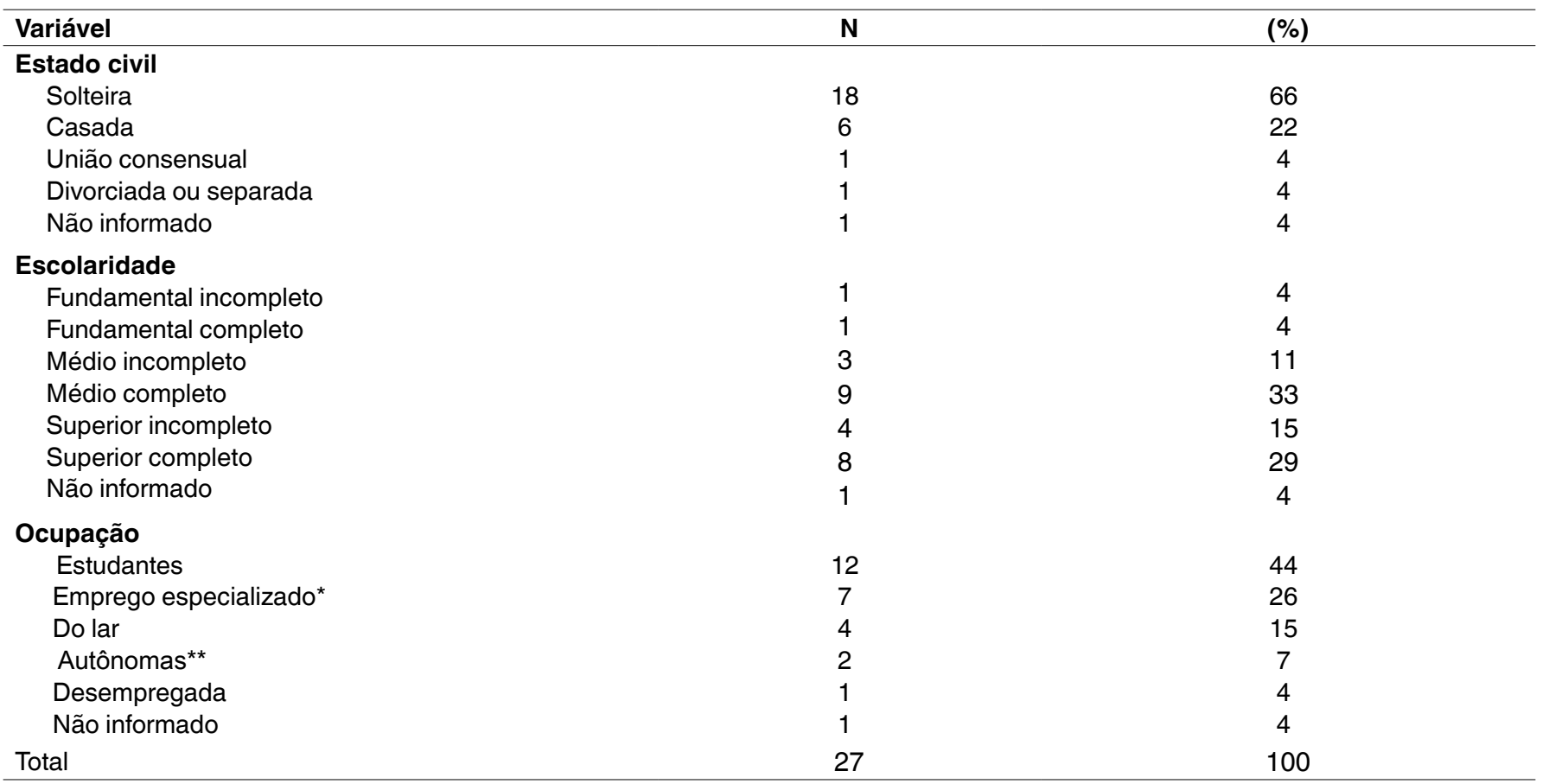

* Farmacêutica, fisioterapeuta, nutricionista, professora e psicóloga

** Esteticista e artesã

O perfil das mulheres que se declararam do lar (Tabela 4) revelou que estas apresentavam uma idade média de 26,5 anos, 70 (72\%) declararam-se casadas ou em união consensual e 48 (49\%) referiram pelo menos ensino médio completo. Foi constatada uma moda de 2,0 e média de 1,9 filhos por mulher, sendo apenas $4(4 \%)$ nulíparas.

Das 246 mulheres do estudo, 195 (79\%) inseriram o dispositivo em João Pessoa e 51 (21\%) nos outros quatro municípios do estado. Em João Pessoa, a faixa etária observada foi de 15 a 50 anos, com idade média de 27 anos, 114 (58\%) mulheres eram casadas ou estavam em união consensual, 117 (60\%) apresentavam pelo menos ensino médio completo, 80 (41\%) eram do lar, enquanto $81(41 \%)$ exerciam atividade remunerada. Já nos demais municípios, a idade variou de 18 a 37 anos, sendo as médias de idade de 26,6 anos. Nesses municípios, 44 (86\%) mulheres eram casadas ou estavam em união consensual, $33(65 \%)$ apresentavam pelo menos ensino médio completo, 17 (33\%) eram do lar, enquanto 29 (57\%) exerciam atividade remunerada. A paridade média encontrada na capital foi de 1,8 filhos, com 166 (85\%) mulheres apresentando um ou mais filhos, já nos demais municípios 49 (96\%) delas referiram ter um ou mais filhos, sendo a média de 1,8 filhos por mulher.

\section{Discussão}

O perfil das mulheres submetidas à inserção do DIU observado no estudo constitui-se de uma amostra com idade média de 27,4 anos e com maioria casada ou em união consensual, com um ou mais filhos, com ensino médio completo e exercendo atividade remunerada. 
Tabela 4 . Perfil das mulheres "do lar" submetidas à inserção de DIU de cobre

$\begin{array}{lcc}\text { Variável } & \mathbf{N} & \text { (\%) } \\ \text { Estado civil } & & 14 \\ \quad \text { Solteira } & 14 & 42 \\ \text { Casada } & 41 & 30 \\ \text { União consensual } & 29 & 3 \\ \text { Divorciada ou separada } & 3 & 10 \\ \quad \text { Não informado } & 11 & \\ \text { Escolaridade } & & 10 \\ \quad \text { Fundamental incompleto } & 10 & 12 \\ \text { Fundamental completo } & 12 & 17 \\ \text { Médio incompleto } & 16 & 42 \\ \text { Médio completo } & 41 & 6 \\ \text { Superior incompleto } & 6 & 1 \\ \text { Superior completo } & 1 & 11 \\ \text { Não informado } & 11 & 4 \\ \text { Paridade } & & 30 \\ 0 & 4 & 39 \\ 1 & 29 & 26 \\ 2 & 38 & 1 \\ \geq 3 & 25 & \text { Moda: } 2 \\ \text { Não informado } & 1 & 100 \\ \text { Paridade Média (anos): } 1,938 & \text { Mediana: } 2 & \\ \text { Total } & 97 & \end{array}$

Os resultados do estudo demonstraram que mulheres de 15 a 50 anos optaram pelo DIU de cobre como método contraceptivo. Aproximadamente, 75\% destas mulheres tinham entre 20 e 34 anos, faixa condizente com o pico do período reprodutivo da mulher. Por outro lado, apenas $8 \%$ das mulheres que inseriram o DIU apresentavam entre 15 e 19 anos, consideradas adolescentes pela OMS.

Alguns fatores podem contribuir para essa porcentagem encontrada. A desaprovação pessoal à atividade sexual na adolescência e a insegurança técnica na prescrição do dispositivo intrauterino de alguns profissionais de saúde constituem barreiras para a difusão desse método contraceptivo, mesmo sendo constatada sua eficácia e segurança nessa faixa etária. ${ }^{8,15-17,28}$ Segundo o Centers for Disease Control and Prevention (CDC), uma maior quantidade de adolescentes usaria métodos mais indicados, caso os médicos fossem melhor informados sobre sua efetividade e segurança. ${ }^{29}$

Estudos em centros especializados mostram que apenas $37 \%$ dos profissionais falam rotineiramente sobre LARCs com adolescentes no aconselhamento reprodutivo inicial. ${ }^{30} \mathrm{Assim}$, as baixas porcentagens de uso de LARCs entre adolescentes podem estar mais associadas aos fatores extrínsecos do que à escolha livre e informada desse grupo sobre esses métodos. ${ }^{31}$

A ocorrência majoritária da iniciação sexual na adolescência e a maior vulnerabilidade dessa população a gravidezes indesejadas e complicações obstétricas são fatores que justificam a necessidade de medidas específicas de planejamento reprodutivo para essa faixa etária. ${ }^{32-34}$ A difusão dos LARCs entre adolescentes vem sendo defendida como alternativa mais eficaz às pílulas anticoncepcionais, por apresentarem eficácia independente da observância da usuária, permitindo que as adolescentes atinjam a idade planejada para própria maternidade, sem gravidezes indesejadas. ${ }^{32,35}$

Observou-se na amostra que $75 \%$ das adolescentes submetidas à inserção do dispositivo apresentavam no mínimo uma gestação prévia. Estudos relacionam a escolha de LARCs por mulheres 
jovens à presença de gravidez prévia. Diante das altas taxas de gravidezes indesejadas nesse grupo, a efetividade dos LARCs funciona como atrativo para essas mulheres, na tentativa de evitar a repetição de uma gestação indesejada. ${ }^{36,37}$

$\mathrm{Na}$ amostra, nota-se também uma redução do número de mulheres submetidas à inserção do DIU, conforme aumento da idade, que pode estar associada à tendência de mulheres mais velhas a apresentarem prole definida e, assim, optarem por métodos contraceptivos definitivos, como a esterilização como também foi observada em estudo realizado em três capitais brasileiras ${ }^{27,38}$.

A maioria das mulheres que inseriram o DIU (64\%) era casada ou estava em união consensual. Estudos mostram relação entre o uso de LARCs e o status do relacionamento, onde mulheres casadas ou em união consensual parecem ser mais propensas a usarem LARCs do que as solteiras. ${ }^{36,39-41}$. A porcentagem encontrada nesse estudo para mulheres casadas ou em união pode expressar uma maior adesão a esse método por esse grupo ou apenas ser uma retrato local do cenário nacional, em que $64 \%$ das mulheres em idade reprodutiva são casadas ou estão em união consensual. ${ }^{4}$

Segundo dados da PNDS de 2006, 50\% das mulheres em idade reprodutiva no Brasil (15 a 49 anos) apresentavam no mínimo ensino fundamental completo. ${ }^{4} \mathrm{~A}$ análise do perfil das mulheres submetidas à inserção do DIU de cobre nesse estudo demonstrou que $68,3 \%$ dessas mulheres apresentavam no mínimo ensino fundamental completo, sendo $61 \%$ a porcentagem das mulheres com pelo menos ensino médio completo.

Tais dados retratam uma maior adesão a esse método contraceptivo pelas mulheres com maior nível de escolaridade, tendência relatada em alguns estudos nacionais e internacionais ${ }^{27,38,42-45}$. Essa relação pode estar associada ao maior acesso dessas mulheres à informação sobre os métodos contraceptivos e sobre a variedade destes, inclusive sobre aqueles mais inovadores, o que lhes confere maior empoderamento na escolha dos mesmos. A maior representatividade desse grupo na adesão ao método pode estar associada à queda das taxas de laqueadura nessas mulheres ao longo dos anos nos Brasil, podendo o DIU representar uma alternativa contraceptiva à escolha da laqueadura por esse grupo. ${ }^{4}$

Em contrapartida, é possível que mulheres com baixo nível de escolaridade sejam mais vulneráveis a incorporar como verdades os mitos disseminados popularmente sobre o DIU. Estudos internacionais relataram proporções expressivas de percepções errôneas sobre o dispositivo intrauterino na APS e demonstraram a interferência direta do aconselhamento profissional preciso na qualificação do conhecimento das mulheres sobre o método, aumentando a probabilidade de adesão ao DIU.46,47

No Brasil, a porcentagem relatada de mulheres em idade reprodutiva exercendo atividade remunerada corresponde a 54,2\%. ${ }^{4}$ No estudo em questão, $44 \%$ das mulheres exerciam alguma atividade remunerada no momento da inserção do DIU, podendo o dispositivo representar para essas mulheres uma tentativa contraceptiva de se garantirem no mercado de trabalho por um intervalo maior de tempo, até atingirem o tempo programado para gestação desejada, oferecendo a elas maior autonomia reprodutiva.

Por outro lado, observou-se uma grande porcentagem de mulheres que se declararam do lar no momento da inserção do DIU. Ao analisar o perfil dessas mulheres, constatou-se que $72 \%$ delas eram casadas ou estavam em união consensual e que, aproximadamente, 95\% delas tinham pelo menos um filho. Sugere-se, então, que a porcentagem expressiva de mulheres que inseriram o DIU nas UBS e declararam-se do lar pode estar intrinsicamente relacionada ao estado civil (estar casada ou em união consensual) e à condição de ter filhos, fatores já associados na literatura à maior propensão a escolha de LARCs, como o DIU. ${ }^{36,39,48,49}$ 
Em relação à paridade das mulheres submetidas à inserção, 11\% eram nulíparas, $87,4 \%$ tinham no mínimo um filho, sendo $72,3 \%$ com um ou dois filhos. Estudos mencionam uma maior probabilidade de uso de LARCs por mulheres que já tiveram filhos e atribuem maiores taxas de adesão aos LARCs por mulheres com um ou dois filhos, em relação àquelas com três ou mais nascimentos, à observância de taxas mais altas de esterilização entre mulheres com maior paridade, com provável prole definida. ${ }^{36,39,48,49}$

A baixa porcentagem de nulíparas submetidas à inserção do DIU no estudo pode refletir a influência de diretrizes antigas na prática atual e o receio dos profissionais quanto à presença de dificuldade de inserção e dor referida pela paciente durante o procedimento. ${ }^{45}$ Atualmente, várias diretrizes asseguram o uso do dispositivo intrauterino à maioria das mulheres, inclusive em adolescentes e nulíparas. ${ }^{15-19}$

Das nulíparas que aderiram ao DIU, $77 \%$ apresentavam pelo menos ensino médio completo, $44 \%$ eram estudantes e $33 \%$ apresentavam atividade remunerada, retratando um grupo de mulheres com boa instrução e inseridas no mercado de trabalho. A escolha de contracepção reversível prolongada por essas mulheres, ainda sem filhos, pode ser um reflexo da autonomia reprodutiva alcançada por elas, mediante a educação e a independência financeira.

No estudo, também foram observadas proporções bem menores de mulheres que inseriram DIU em UBS fora do município de João Pessoa. Tais resultados podem estar associados a barreiras organizacionais para inserção do DIU na APS desses municípios ou a barreiras individuais para adesão ao dispositivo pelas mulheres residentes fora da capital do estado. ${ }^{27}$

Foram consideradas como limitações do estudo a ausência de informações sobre as características avaliadas de algumas mulheres nos prontuários médicos e nos registros do procedimento de inserção. Entre os dados com maior perda, estão o estado civil (9\%) e a escolaridade (11\%) pois algumas UBS não os incluío nos seus primeiros registros limitando uma análise da totalidade dos dados. Outra limitação foi a falta de alguns dados importantes no perfil dessas mulheres como raça, história obstétrica e historia de gestações indesejadas, além da quantidade escassa de literatura sobre as características das mulheres que aderem ao DIU como método contraceptivo no Brasil e no mundo no contexto da APS para uma melhor análise comparativa.

Cabe ao projeto de extensão apresentar à equipe os critérios de elegibilidade e as etapas do treinamento e é de responsabilidade dos profissionais fazerem a oferta e seleção das mulheres que irão inserir o DIU. Diante disso, o projeto interfere parcialmente no resultado do estudo quanto ao perfil das mulheres que inseriram o DIU no momento que discute os critérios de elegibilidade, no entanto, uma ação pontual não é suficiente para atrair alguns perfis de mulheres.

No que tange ainda às limitações, os achados correspondem a dados de mulheres que habitam na capital e em três municípios da região metropolitana com menos de 50000 habitantes, o que corresponde ao perfil da maioria dos municípios paraibanos. No entanto deve-se entender a limitação do estudo frente a diversidade geográfica e cultural da população brasileira.

\section{Conclusão}

A análise do perfil das mulheres submetidas à inserção do DIU nas trezes UBS participantes do projeto de treinamento do DIU mostrou predomínio de mulheres de 20 a 29 anos, casadas ou em união consensual, com boa escolaridade, exercendo atividade remunerada e com um ou mais filhos. Em 
contrapartida, verificaram-se baixas porcentagens de adolescentes, mulheres de baixa escolaridade e nulíparas na amostra.

Conhecer as características das mulheres que optam pelo DIU como método contraceptivo permite traçar estratégias específicas para romper barreiras organizacionais e individuais para adesão ao DIU. O perfil das mulheres no estudo aponta a necessidade de gestores construírem protocolos baseados em evidências, elaborarem estratégias para alcançar os grupos específicos com divulgação e linguagem apropriada, assim como realizar treinamento para os profissionais de saúde enfatizando os grupos elegíveis mas com pouca adesão. Da mesma forma, os dados mostram a importância dos profissionais de realizarem educação permanente e ter condutas baseadas nos critérios de elegibilidade.

O resultado aponta a necessidade de futuras pesquisas na APS que possam aprofundar o conhecimento de outros aspectos relacionados às mulheres como o estudo da dor, reações adversas e complicações que possam trazer segurança da oferta e na inserção dentro das UBS para ampliar o perfil das mulheres que inserem o DIU.

\section{Conflitos de interese}

Os autores declaram o presente trabalho é original, não é resultante de plágio, não foi publicado e não está sendo considerado para publicação em outra revista, quer seja no formato impresso ou no eletrônico. Declaram ainda que não apresenta conflitos de interesse pessoais, empresariais ou governamentais que poderiam comprometer a obtenção e divulgação dos resultados bem como a discussão e conclusão do estudo.

\section{Contribuição dos autores}

Todos os autores participaram das diversas etapas do estudo, conforme descrito a seguir:

Concepção do estudo: IGFM, DSB, MEMPR, RDG

Delineamento do estudo: IGFM, DSB, RSS, AJMN

Aquisição dos dados: IGFM, DSB, MEMPR, RDG, PSR

Análise dos dados: IGFM, DSB, RSS, AJMN

Interpretação dos dados: IGFM, DSB, AJMN, PSR

Elaboração do rascunho do trabalho: IGFM, MEMPR, RDG, PSR

Revisão crítica do conteúdo: DSB, RSS, AJMN

Aprovaram da versão final a ser publicada: IGFM, DSB, MEMPR, RDG, RSS, AJMN, PSR

Todos os autores concordaram em prestar contas sobre todos os aspectos do trabalho.

\section{Referências}

1. Ministério da Saúde (BR), Secretaria de Atenção à Saúde, Departamento de Atenção Básica. Saúde sexual e saúde reprodutiva. Brasília: Ministério da Saúde, 2013.

2. Piovesan F. Temas de direitos humanos. 9.ed. São Paulo: Saraiva, 2016.

3. Bearak J, Popinchalk A, Alkema L, et al. Global, regional, and sub-regional trends in unintended pregnancy and its outcomes from 1990 to 2014: estimates from a Bayesian hierarchical model. Lancet Glob Health. 2018; 6(4): e380-e389. DOI: https://doi.org/10.1016/ S2214-109X(18)30029-9

4. Ministério da Saúde (BR). Pesquisa Nacional de Demografia e Saúde da Criança e da Mulher - PNDS 2006: dimensões do processo reprodutivo e da saúde da criança. Brasília: Ministério da Saúde, 2009. 
5. Viellas EF, Domingues RMSM, Dias MAB et al. Prenatal care; maternal and child health; maternal-child health services. Cad Saude Publica. 2014; 30 (Suppl 1): S1-S15.

6. Cleland J, Conde-Agudelo A, Peterson H, Ross J, Tsui A. Contraception and health. Lancet. 2012; 380(9837): 149-156. PMID: 22784533 DOI: https://doi.org/10.1016/S0140-6736(12)60609-6

7. Espey E, Ogburn T. Long-acting reversible contraceptives: intrauterine devices and the contraceptive implant. Obstet Gynecol. 2011; 117(3): 705-719 PMID: 21343774 DOI: https://doi.org/10.1097/AOG.0b013e31820ce2f0

8.Winner B, Peipert JF, Zhao Q, et al. Effectiveness of long-acting reversible contraception. Engl J Med. 2012; 366(21): 1998-2007. DOI: https://doi.org/10.1056/NEJMoa1110855

9.Blumenthal PD, Voedisch A, Gemzell-Danielsson K. Strategies to prevent unintended pregnancy: increasing use of longacting reversible contraception. Hum Reprod Update. 2011; 17(1):121-137. DOI: https://doi.org/10.1093/humupd/dmq026

10. Trussell J, Hassan F, Lowin J, Law A, Filonenko A. Achieving cost-neutrality with long-acting reversible contraceptive methods. Contraception. 2015; 91(1): 49-56. PMID: 25282161 DOI: https://doi.org/10.1016/j.contraception.2014.08.011

11. Rivera R, Yacobson I, Grimes D. The mechanism of action of hormonal contraceptives and intrauterine contraceptive devices. Am J Obstet Gynecol. 1999; 181:1263-9. PMID: 10561657 DOI: https://doi.org/10.1016/S0002-9378(99)70120-1

12.Ortiz ME, Croxatto HB. Copper-T intrauterine device and levonorgestrel intrauterine system: biological bases of their mechanism of action. Contraception. 2007; 75: S16-30 PMID: 17531610 DOI: https://doi.org/10.1016/j.contraception.2007.01.020

13. Trussell J. Contraceptive failure in the United States. Contraception. 2011; 83(5): 397-404. DOI: https://doi.org/10.1016/j. contraception.2011.01.021

14. Girum T, Wasie A. Return of fertility after discontinuation of contraception: a systematic review and meta-analysis. Contracept Reprod Med. 2018; 3 (1): 9. PMID: 30062044 DOI: https://doi.org/10.1186/s40834-018-0064-y

15. American College of Obstetricians and Gynecologists. ACOG Practice Bulletin No. 121: Long-acting reversible contraception: implants and intrauterine devices. Obstet Gynecol. 2011; 118(1): 184-196. DOI: https://doi.org/10.1097/AOG.0b013e318227f05e

16. Diedrich JT, Klein DA, Peipert JF. Long-acting reversible contraception in adolescents: a systematic review and meta-analysis. Am J Obst Ginecol. 2017; 216(4): 364-e1. DOI: https://doi.org/10.1016/j.ajog.2016.12.024

17. Centers for Disease Control and Prevention. U.S. Medical eligibility criteria for contraceptive use, 2010. MMWR Recomm Rep. 2010; 59(RR-4): 1-86. PMID: 20559203

18. Shaamash AH, Sayed GH, Hussien MM, Shaaban MM. A comparative study of the levonorgestrel-releasing intrauterine system Mirena versus the Copper T380A intrauterine device during lactation: breast-feeding performance, infant growth and infant development. Contraception. 2005; 72(5): 346-351. PMID: 16246660 DOI: https://doi.org/10.1016/j.contraception.2005.04.004

19. Campbell SJ, Cropsey KL, Matthews CA. Intrauterine device use in a high-risk population: experience from an urban university clinic. Am J Obstet Gynecol. 2007; 197(2): 193.e1-193.e6. DOI: https://doi.org/10.1016/j.ajog.2007.04.028

20. Steenland MW, Tepper NK, Curtis KM, Kapp N. Intrauterine contraceptive insertion postabortion: a systematic review. Contraception 2011; 84: 447-64. PMID: 22018119 DOI: https://doi.org/10.1016/j.contraception.2011.03.007

21. Washington $\mathrm{Cl}$, Jamshidi R, Thung SF, Nayeri UA, Caughey AB, Werner EF. Timing of postpartum intrauterine device placement: a cost-effectiveness analysis. Fertil Steril. 2015; 103: 131-7. PMID: 25439838 DOI: https://doi.org/10.1016/j.fertnstert.2014.09.032

22. Grimes DA, Lopez LM, Manion C, Schulz KF. Cochrane systematic reviews of IUD trials: lessons learned. Contraception. 2007; 75(6 Suppl): S55-9. PMID: 17531618 DOI: https://doi.org/10.1016/j.contraception.2006.12.004

23. O'Neil-Callahan M, Peipert JF, Zhao Q, Madden T, Secura G. Twenty-four-month continuation of reversible contraception. Obstet Gynecol. 2013; 122: 1083-91. PMID: 24104781

24. Diedrich JT, Madden T, Zhao Q, Peipert JF. Long-term utilization and continuation of intrauterine devices. Am J Obstet Gynecol. 2015; 213(6): 822.e1-822.e6. PMID: 26409157 DOI: https://doi.org/10.1016/j.ajog.2015.08.077

25. Gonzaga, VAS, Borges ALV, Santos OAD, Rosa PLFS, Gonçalves RFS. Barreiras organizacionais para disponibilização e inserção do dispositivo intrauterino nos serviços de atenção básica à saúde. Rev Esc Enferm USP. 2017; 51, e03270-e03270. DOI: https://doi. org/10.1590/s1980-220x2016046803270

26. Svanemyr J, Chandra-Mouli V, Christiansen CS, Mbizvo M. Preventing child marriages: first international day of the girl child "my life, my right, end child marriage". Reprod Health. 2012; 9:31. PMID: 23163964 DOI: https://doi.org/10.1186/1742-4755-9-31 
27. Borges ALV, Araújo KS, Santos AO, Gonçalves RFS, Fujimori E, Divino EA. Knowledge about the intrauterine device and interest in using it among women users of primary care services. Rev. Latino-Am. Enfermagem. 2020; 28: e3232. DOI: https://doi.org/10.1590/15188345.3140 .3232

28. Lowes L, Eddy D, Channon S, McNamara R, Robling M, Gregory JW. The experience of living with type 1 diabetes and attending clinic from the perception of children, adolescents and carers: analysis of qualitative data from the DEPICTED study. J Pediatr Nurs. 2015; 30(1): 54-62. PMID: 25308399 DOI: https://doi.org/10.1016/j.pedn.2014.09.006

29. Kavanaugh ML, Jerman J, Ethier K, Moskosky S. Meeting the contraceptive needs of teen and Young adults:youth-friendly and long-acting reversible contraceptive services in U.S. family planning facilities. J Adolesc Health. 2013; 52: 284-92. DOI: https://doi.org/10.1016/j. jadohealth.2012.10.276

30. Hubacher D. Long-acting reversible contraception acceptability and satisfaction is high among adolescents. BMJ Evidence-Based Medicine. 2017; 22(6): 228-229. DOI: https://doi.org/10.1136/ebmed-2017-110768

31. Borges ALV, Fujimori E, Kuschnir MCC, Chofakian CBDN, Moraes AJPD, Azevedo GD, et al. ERICA: sexual initiation and contraception in Brazilian adolescents. Rev saúde públ. 2016; 50: 15s. DOI: https://doi.org/10.1590/s01518-8787.2016050006686

32. Vasilenko AS, Kugler KC, Rice CE. Timing of first sexual intercourse and young adult health outcomes. J Adol Health. 2016 ; 59(3):291-7. DOI: https://doi.org/10.1016/j.jadohealth.2016.04.019

33. Yazlle, MEHD. Gravidez na adolescência. Rev. Bras. Ginecol Obstet. 2006; 28(8): 443-445.

34. Teal SB, Romer, SE. Awareness of long-acting reversible contraception among teens and young adults. J Adolesc Health. 2013; 52(4): S35-S39. DOI: https://doi.org/10.1016/j.jadohealth.2013.01.013

35. Coombe J, Harris ML, Loxton D. Who uses long-acting reversible contraception? Profile of LARC users in the CUPID cohort. Sex Reprod Healthc. 2017; 11: 19-24. PMID: 28159123 DOI: https://doi.org/10.1016/j.srhc.2016.09.003

36. Herbert D, Harris M, Loxton D, Lucke J. Contraceptive use and unintended pregnancy among 18-23-year-old women in Australia: the first findings of the CUPID study. Eur J Contracept Reprod Health Care. 2013; 18:S78-9.

37. de Souza Barros J V, Wong LLR. Prevalência, conhecimento e tipos de métodos contraceptivos utilizados pelas mulheres, segundo o tipo de união: um estudo para Brasil e México. Anais. 2016; 1-20.

38. Kavanaugh ML, Jerman J, Hubacher D, Kost K, Finer LB. Characteristics of women in the United States who use long-acting reversible contraceptive methods. Obstet Gynecol.2011;117(6):1349-57.PMID:21606745 DOI:https://doi.org/10.1097/AOG.0b013e31821c47c9

39. Gray E, Arunachalam D. Patterns of contraceptive use. In: Heard G, Arunachalam D, editors. Family formation in 21 st century Australia. Dordrecht: Springer. 2015; p.123-40.

40. Haimovich S. Profile of long-acting reversible contraception users in Europe. Eur J Contracept Reprod Health Care. 2009; 14(3): 187195. PMID: 19565416 DOI: https://doi.org/10.1080/13625180902741436

41. Regianini HA. Perfil das pacientes submetidas à inserção de dispositivo intrauterino no Centro de Saúde dos Ingleses. Florianópolis: Universidade Federal de Santa Catarina; 2009.

42. Vieira EM, Badiani R, Dal Fabbro AL, Rodrigues Junior AL. Características do uso de métodos anticoncepcionais no Estado de São Paulo. Ver Saud Publ. 2002; 36: 263-270. DOI: https://doi.org/10.1590/S0034-89102002000300002

43. Abasiattai AM, Bassey EA, Udoma EJ. Profile of intrauterine contraceptive device acceptors at the university of Uyo teaching hospital, Uyo, Nigeria. Ann Afr Med. 2008; 7:1-5. PMID: 18702241 DOI: https://doi.org/10.4103/1596-3519.55692

44. Xu X, Macaluso M, Frost J, Anderson JE, Curtis K, Grosse SD. Characteristics of users of intrauterine devices and other reversible contraceptive methods in the United States. Fertil Steril. 2011; 96(5): 1138-1144. PMID: 21917255 DOI: https://doi.org/10.1016/j. fertnstert.2011.08.019

45. Callegari LS, Parisi, SM, Schwarz EB. Perceptions of intrauterine contraception among women seeking primary care. Contraception. 2013; 88(2), 269-274. DOI: https://doi.org/10.1016/j.contraception.2013.02.004

46. Hladky KJ, Allsworth JE, Madden T, Secura GM, Peipert JF. Women's knowledge about intrauterine contraception. Obstet Gynecol. 2011;117:48-54 PMID: 21173643 DOI: https://doi.org/10.1097/AOG.0b013e318202b4c9

47. Chan LM, Westhoff CL. Tubal sterilization trends in the United States. Fertil Steril. 2010; 94: 1- 6. PMID: 20497790 DOI: https://doi. org/10.1016/j.fertnstert.2010.03.029

48. Hillis SD, Marchbanks PA, Tylor LR, Peterson HB. Poststerilization regret: findings from the United States Collaborative Review of Sterilization. Obstet Gynecol. 1999; 93: 889-95. PMID: 10362150 DOI: https://doi.org/10.1097/00006250-199906000-00001 